\title{
MONOTONICITY PROPERTIES OF MINIMIZERS AND RELAXATION FOR AUTONOMOUS VARIATIONAL PROBLEMS
}

\author{
Giovanni Cupini $^{1}$ And Cristina Marcelli ${ }^{2}$
}

\begin{abstract}
We consider the following classical autonomous variational problem

$$
\operatorname{minimize}\left\{F(v)=\int_{a}^{b} f\left(v(x), v^{\prime}(x)\right) \mathrm{d} x: v \in A C([a, b]), v(a)=\alpha, v(b)=\beta\right\},
$$

where the Lagrangian $f$ is possibly neither continuous, nor convex, nor coercive. We prove a monotonicity property of the minimizers stating that they satisfy the maximum principle or the minimum one. By virtue of such a property, applying recent results concerning constrained variational problems, we derive a relaxation theorem, the DuBois-Reymond necessary condition and some existence or non-existence criteria.
\end{abstract}

Mathematics Subject Classification. 49K05, 49J05.

Received November 20, 2008.

Published online March 24, 2010.

\section{INTRODUCTION}

This paper is devoted to the study of various aspects of the classical autonomous variational problem

$$
\operatorname{minimize}\left\{F(v)=\int_{a}^{b} f\left(v(x), v^{\prime}(x)\right) \mathrm{d} x: v \in \Upsilon\right\}
$$

where $\Upsilon$ is the class of absolutely continuous functions $u:[a, b] \rightarrow I, u(a)=\alpha, u(b)=\beta$ and $I$ is a bounded or unbounded real interval including $\alpha$ and $\beta$. The main feature is that the Lagrangian $f: I \times \mathbb{R} \rightarrow[0,+\infty)$, $f=f(s, z)$, is quite general, since $f$ may be discontinuous and $f(s, \cdot)$ is not assumed to be convex or coercive. As it is well known, the lack of convexity and coercivity does not allow the use of the classical direct methods of the Calculus of Variations and the variational problem can have no solution.

Keywords and phrases. Nonconvex variational problems, autonomous variational problems, existence of minimizers, DuBoisReymond necessary condition, relaxation.

1 Dipartimento di Matematica, Università di Bologna, Piazza di Porta S.Donato 5, 40126 Bologna, Italy.

giovanni.cupini@unibo.it

2 Dipartimento di Scienze Matematiche, Università Politecnica delle Marche, Via Brecce Bianche, 60131 Ancona, Italy.

marcelli@dipmat.univpm.it 
The mathematical literature on this context is very rich; different classes of Lagrangians have been considered by many authors and in various settings of applicability. Among the many related papers, for the case of $f$ coercive but not convex we quote [21,25] ( $f$ smooth depending also on $x$ ), [17], see also [22], where, under very weak regularity assumptions, Lagrangians of sum-type are considered. Concerning the case when $f$ is noncoercive, but convex, we indicate $[8,11]$ where the existence of Lipschitz continuous minimizers is established under different sets of assumptions, admitting some cases of linear growth for the integrand and, in case of [11], even some cases in which $f$ does not exhibit such a growth. We also mention $[3-5,19]$ for the case of noncoercive, nonautonomous Lagrangians. The existence of the minimum for a class of nonconvex $f$ has been studied in [6] under a growth assumption similar to that considered in $[8,9]$, while the relaxation and the lipschitzianity of solutions are studied in [7].

Despite the intensive researches on this topic, the theory does not seem sufficiently developed yet and many questions are still open problems, concerning the necessary conditions for the optimality, the relaxation problem, and the sufficient conditions to the existence of minimizers in the nonconvex and noncoercive case. Our aim in this paper is to provide some answers to the previous questions.

Our starting point and main tool is the study of the monotonicity properties of the minimizers of $F$ (when they exist). It is well-known (see [18]) that if $f(s, 0)<f(s, z)$ for every $z \neq 0$, and $s \mapsto f(s, 0)$ is increasing, then any possible minimizer satisfies the maximum principle in any subinterval, i.e., there exists a point $x_{0} \in[a, b]$ such that the minimizer is decreasing in $\left[a, x_{0}\right]$, increasing in $\left[x_{0}, b\right]$. This was recently improved in [12], Theorem 3.1, where the strict inequality $f(s, 0)<f(s, z)$ was replaced by the weak one. Moreover, under a certain monotonicity condition on $s \mapsto f(s, 0)$, it was shown that the minimizers satisfy the maximum principle or the minimum one.

A study of the monotonicity properties of the minimizers was also carried out in $[17,22]$ for proving the existence of the minimum in the case of integrands having the sum structure $f(s, z)=g(s)+h(z)$ with $h$ coercive but nonconvex, without any monotonicity condition on the term $g$. Subsequently, this investigation was extended in [24] for coercive integrands having a general structure.

In this paper, with no coercivity hypothesis, we prove (see Thm. 3.1) that under the sole assumptions that $f(s, z)$ is Borel-measurable, $s \mapsto f(s, 0)$ is lower semicontinuous and $f(s, 0)=f^{* *}(s, 0)$, then the competition set $\Upsilon$ can be restricted to the subclass collecting those trajectories such that for some interval $\left[\tau_{1}, \tau_{2}\right] \subseteq[a, b]$ they are constant in $\left[\tau_{1}, \tau_{2}\right]$, strictly monotone (and with a.e. non-vanishing derivative) in $\left[a, \tau_{1}\right]$ and in $\left[\tau_{2}, b\right]$.

Such a monotonicity property allows to use results known for constrained problems (see [20]). Indeed, a first application is the DuBois-Reymond necessary condition (see Thm. 5.1), which in our non-smooth setting assumes the form of a differential inclusion:

$$
f\left(u(x), u^{\prime}(x)\right)-c \in u^{\prime}(x) \partial f\left(u(x), u^{\prime}(x)\right) \quad \text { a.e. in }(a, b)
$$

where $\partial f(s, \cdot)$ denotes the subdifferential in the sense of Convex Analysis, even if the integrand is nonconvex in general. Notice that as an immediate consequence one derives that if $u$ is a minimizer, then $f\left(u(x), u^{\prime}(x)\right)=$ $f^{* *}\left(u(x), u^{\prime}(x)\right)$ a.e. in $(a, b)$.

The (DBR) condition was obtained also in [1] for integrands $f: \mathbb{R}^{n} \times \mathbb{R}^{n} \rightarrow[0,+\infty]$, in the convex case, see also [14] for a related result in the nonconvex, coercive framework.

A further relevant property consists in an upper bound for the constant $c$ in the condition (DBR), that is

$$
c \leq \min _{s \in u([a, b])} f(s, 0)
$$

Thanks to this limitation on the constant $c$ we are able to derive a non-existence result (see Prop. 5.2), stating that if

$$
\text { ess } \sup _{s \in[\alpha, \beta]} \liminf _{|z| \rightarrow+\infty} \inf _{\xi \in \partial f^{* *}(s, z)}\left(f^{* *}(s, z)-z \xi\right)>\min _{s \in[\alpha, \beta]} f(s, 0)
$$

then the minimum does not exist. In the recent paper [12] analogous results were proved to those described in Section 5 , but under a certain monotonicity assumption on $f(\cdot, 0)$. 
The monotonicity property of minimizers allows us also to treat the question of the relaxation. Indeed, we prove (see Thm. 4.2) that if $f^{* *}(s, 0)$ attains its minimum value only on a set of $s$ of null measure, or, alternatively, if the detachment set $\Delta_{s}:=\left\{z: f^{* *}(s, z)<f(s, z)\right\}$ does not contain any interval $(0, \delta)$ or $(-\delta, 0)$ (assumption (4.2)), then $F$ admits minimum provided that the relaxed functional $F^{* *}$ admits a minimizer $v$ satisfying

$$
v^{\prime}(x) \in \operatorname{co}\left(\mathbb{R} \backslash \Delta_{v(x)}\right) \quad \text { for a.e. } x \in(a, b) .
$$

Finally, we prove that assumption (4.2) can be removed in the coercive case (see Thm. 4.3). Such a result assumes a very simple form when the integrand has the affine-type structure

$$
f(s, z)=g(s)+\gamma(s) h(z) .
$$

Indeed, we show that if $g$ is lower semicontinuous, non-negative, $\gamma$ is continuous with inf $\gamma>0, h$ is Borelmeasurable, coercive, with $h(0)=h^{* *}(0)$, then $F$ admits minimum (see Cor. 4.4). This result extends those proved in $[17,22]$.

Our paper is organized as follows: in the next section we present the notations and some preliminary results, as a reparametrization procedure, basic tool for the study of the monotonicity properties of the minimizers. In Section 3 we study the monotonicity properties of the minimizers: the main result, Theorem 3.1, states that the minimizers may be searched among the functions satisfying the maximum/minimum principle. Section 4 is devoted to relaxation results, obtained in the general case (Thm. 4.2) and, in the coercive case, under weaker assumptions (Thm. 4.3 and Cor. 4.4 for affine-type Lagrangians). Eventually, in Section 5 we claim that the DuBois-Reymond is a necessary condition for the optimality (Thm. 5.1) and we state a nonexistence result (Prop. 5.2) with some examples of applicability.

\section{Notations AND PRELIMINARY RESUlts}

Let us consider the following autonomous minimization problem

$$
\operatorname{minimize}\left\{F(v)=\int_{a}^{b} f\left(v(x), v^{\prime}(x)\right) \mathrm{d} x: v \in \Upsilon\right\},
$$

where

$$
\Upsilon:=\left\{v \in W^{1,1}(a, b): v(a)=\alpha, v(b)=\beta, v(x) \in I\right\},
$$

$I \subseteq \mathbb{R}$ is a generic interval, possibly unbounded, including $\alpha, \beta$, and $f: I \times \mathbb{R} \rightarrow[0,+\infty)$ is a Borel-measurable function with $f(\cdot, 0)$ lower semicontinuous. Note that $f(s, \cdot)$ is not assumed to be convex or coercive.

By $\partial f(s, z)$ we denote the classical subdifferential in the sense of Convex Analysis of $f(s, \cdot)$ at $z$, that is

$$
\partial f(s, z):=\{\xi \in \mathbb{R}: f(s, w)-f(s, z) \geq \xi(w-z) \text { for every } w \in \mathbb{R}\} .
$$

As usual, $f^{* *}$ denotes the convex envelope of $f$ with respect to the second variable, i.e., fixed $s \in I, f^{* *}(s, \cdot)$ is the largest convex function lower than $f(s, \cdot)$.

As it was put in evidence in [17], a crucial assumption for the existence of the minimum in the nonconvex case is the following

$$
f(s, 0)=f^{* *}(s, 0) \quad \text { for every } s \in I .
$$

In the sequel we will also consider the relaxed problem

$$
\operatorname{minimize}\left\{F^{* *}(v)=\int_{a}^{b} f^{* *}\left(v(x), v^{\prime}(x)\right) \mathrm{d} x: v \in \Upsilon\right\} .
$$

Notice that $F^{* *}$ is well defined as shown in the following result. 
Lemma 2.1. Let $f: I \times \mathbb{R} \rightarrow[0,+\infty)$ be a Borel-measurable function satisfying (2.2). Then, the function $s \mapsto f^{* *}(s, z)$ is Lebesgue-measurable for every $z \in \mathbb{R}$ and the function $x \mapsto f^{* *}\left(v(x)\right.$, $\left.v^{\prime}(x)\right)$ is Lebesguemeasurable for every $v \in W_{\mathrm{loc}}^{1,1}(a, b)$.

Proof. Fixed $z \in \mathbb{R}$, define $g_{z}:[0,1] \times I \times \mathbb{R} \rightarrow[0,+\infty)$,

$$
g_{z}(\alpha, s, w):= \begin{cases}\alpha f(s, w)+(1-\alpha) f\left(s, \frac{z-\alpha w}{1-\alpha}\right) & \text { if } 0 \leq \alpha<1 \\ f(s, w) & \text { if } \alpha=1\end{cases}
$$

Since $f$ is Borel measurable, then $g_{z}$ is Borel measurable, too. Moreover, see e.g. [13], Theorem 2.35,

$$
\begin{aligned}
f^{* *}(s, z) & =\inf \left\{\alpha f\left(s, w_{1}\right)+(1-\alpha) f\left(s, w_{2}\right): 0 \leq \alpha \leq 1, w_{1}, w_{2} \in \mathbb{R}, \alpha w_{1}+(1-\alpha) w_{2}=z\right\} \\
& =\inf \left\{g_{z}(\alpha, s, w): 0 \leq \alpha \leq 1, w \in \mathbb{R}\right\} .
\end{aligned}
$$

In order to show that $s \mapsto f^{* *}(s, z)$ is Lebesgue-measurable, let us prove that the set

$$
A_{k}:=\left\{s \in I: \inf _{0 \leq \alpha \leq 1, w \in \mathbb{R}} g_{z}(\alpha, s, w)<k\right\}
$$

is Lebesgue-measurable for every $k \in \mathbb{R}$. To this end, define

$$
B_{k}:=\left\{(\alpha, s, w) \in[0,1] \times I \times \mathbb{R}: g_{z}(\alpha, s, w)<k\right\}
$$

and notice that $A_{k}=\pi\left(B_{k}\right)$, where $\pi:[0,1] \times I \times \mathbb{R} \rightarrow I$ is the projection $\pi(\alpha, s, w)=s$. Hence, since $B_{k}$ is Borel-measurable and $\pi$ is a continuous function, then $A_{k}$ is Lebesgue-measurable (see e.g. [2], Cor. 1.10.9) and we get the claim.

Finally, since $z \mapsto f^{* *}(s, z)$ is continuous and $s \mapsto f^{* *}(s, 0)=f(s, 0)$ is a Borel function, then by [15], Lemma 3, we deduce that the function $x \mapsto f^{* *}\left(v(x), v^{\prime}(x)\right)$ is Lebesgue-measurable for every $v \in W_{\text {loc }}^{1,1}(a, b)$.

The next lemma, proved in a slight stronger version in [12], Lemma 2.1, states that under condition (2.2) we can also assume, without loss of generality, that

$$
f(s, 0)=\min _{z \in \mathbb{R}} f(s, z) \quad \text { for every } s \in I .
$$

Lemma 2.2. Let $f: I \times \mathbb{R} \rightarrow[0,+\infty)$ be a Borel-measurable function satisfying (2.2) and such that

$$
\text { there exists a Borel-measurable selection } g(s) \in \partial f^{* *}(s, 0) \text { with } g \in L_{\mathrm{loc}}^{\infty}(I) \text {. }
$$

Then there exists a Borel-measurable function $\tilde{f}: I \times \mathbb{R} \rightarrow[0,+\infty)$ with the following properties:

(a) $f(s, 0)=\tilde{f}(s, 0)=(\tilde{f})^{* *}(s, 0)$ for every $s \in I$;

(b) $\tilde{f}(s, 0)=\min _{z \in \mathbb{R}} \tilde{f}(s, z)$ for every $s \in I$;

(c) there exists $k \in \mathbb{R}$ such that

$$
F(u)=\tilde{F}(u)+k \quad \text { for every } u \in \Upsilon
$$

where $\tilde{F}(u)$ stands for $\int_{a}^{b} \tilde{f}\left(u(x), u^{\prime}(x)\right) \mathrm{d} x$.

Moreover, if $f$ is a lower semicontinuous function and $g$ is continuous, then $\tilde{f}$ is lower semicontinuous too.

Proof. It is easy to show that the function $\tilde{f}(s, z):=f(s, z)-g(s) z$ satisfies the properties (a) and (b) in the statement. To prove (c), consider $u \in \Upsilon$ and define $G(t)=\int_{\alpha}^{t} g(s) \mathrm{d} s$. Then $G \in W_{\mathrm{loc}}^{1, \infty}(I), G \circ u \in A C([a, b])$ and by the De La Vallée-Poussin lemma the chain rule $(G \circ u)^{\prime}(x)=G^{\prime}(u(x)) u^{\prime}(x)$ holds for a.e. $x \in(a, b)$. Moreover, 
there exists a negligible set $N \subset I$ such that $G^{\prime}(s)=g(s)$ for every $s \in I \backslash N$. Let $B$ be a Borel negligible set such that $N \subseteq B$. We have that $u^{-1}(B)$ is Lebesgue-measurable and $u^{\prime}(x)=0$ for a.e. $x \in u^{-1}(B)$. Therefore

$$
\int_{a}^{b} g(u(x)) u^{\prime}(x) \mathrm{d} x=\int_{a}^{b} G^{\prime}(u(x)) u^{\prime}(x) \mathrm{d} x=\int_{a}^{b}(G \circ u)^{\prime}(x) \mathrm{d} x=G(\beta)-G(\alpha) .
$$

Remark 2.3. Replacing the Borel measurability of $f$ with

$$
x \mapsto f\left(v(x), v^{\prime}(x)\right) \quad \text { is Lebesgue-measurable for every } v \in \Upsilon,
$$

in the assumptions of Lemma 2.2, then the assertion still holds, with $\tilde{f}$ not necessarily Borel-measurable, but satisfying (2.6).

Remark 2.4. As for condition (2.5), notice that it is satisfied when $f^{* *}(\cdot, z)$ is Borel-measurable for all $z$, and $f^{* *}(\cdot, \pm 1) \in L_{\mathrm{loc}}^{\infty}(I)$. Indeed, the function

$$
g(s):=\inf _{n} n\left[f^{* *}\left(s, \frac{1}{n}\right)-f^{* *}(s, 0)\right]
$$

is Borel-measurable and it is easy to prove that $g(s) \in \partial f^{* *}(s, 0)$. Moreover, note that

$$
-f^{* *}(s,-1) \leq f^{* *}(s, 0)-f^{* *}(s,-1) \leq g(s) \leq f^{* *}(s, 1)-f^{* *}(s, 0) \leq f^{* *}(s, 1)
$$

and we conclude that $g \in L_{\mathrm{loc}}^{\infty}(I)$.

One of the aims of the present paper is to study the monotonicity properties of the minimizers. A first step to obtain monotone trajectories is the following lemma proved in [12].

Lemma 2.5 ([12], Lem. 3.2). Let $u:[c, d] \rightarrow \mathbb{R}$ be an absolutely continuous function such that $u(c) \leq u(x)$ (respectively $u(c) \geq u(x)$ ) for every $x \in[c, d]$. Then, there exists an increasing (a decreasing) absolutely continuous function $v:[c, d] \rightarrow \mathbb{R}$ such that $v(c)=u(c), v(d)=u(d), v(x) \leq u(x)$ for every $x \in[c, d]$, and

$$
v(x)=u(x) \text { and } v^{\prime}(x)=u^{\prime}(x) \quad \text { for a.e. } x \text { such that there exists } v^{\prime}(x) \neq 0 .
$$

In the sequel we often will need to manipulate monotone functions. To this purpose, we now recall a reparametrization introduced in [20], which allows to unify the constant tracts of a monotone function, as follows.

Let $v:[c, d] \rightarrow[\gamma, \delta]$ be a monotone increasing, absolutely continuous function, with $v([c, d])=[\gamma, \delta] \subseteq I$, and define

$$
A_{v}:=\left\{x: \exists v^{\prime}(x) \neq 0\right\} \quad \text { and } \quad B_{v}:=\left\{x: \exists v^{\prime}(x)=0\right\} .
$$

Consider the function $\chi_{v}^{+}:[\gamma, \delta] \rightarrow[c, d]$ defined by

$$
\chi_{v}^{+}(s):=c+\int_{\gamma}^{s}\left(w_{v}^{+}\right)^{\prime}(\sigma) \mathrm{d} \sigma, \quad \text { where } w_{v}^{+}(s):=\min \{x \in[c, d]: v(x)=s\} .
$$

Moreover, chosen

$$
s_{0} \in\left\{\tau \in[\gamma, \delta]: f(\tau, 0)=\min _{s \in[\gamma, \delta]} f(s, 0)\right\},
$$

let us consider the function $\Psi_{v}^{+}:[c, d] \rightarrow[\gamma, \delta]$ defined by

$$
\Psi_{v}^{+}(x):= \begin{cases}\left(\chi_{v}^{+}\right)^{-1}(x) & \text { if } c \leq x \leq \chi_{v}^{+}\left(s_{0}\right) \\ s_{0} & \text { if } \chi_{v}^{+}\left(s_{0}\right) \leq x \leq \chi_{v}^{+}\left(s_{0}\right)+d-\chi_{v}^{+}(\delta) \\ \left(\chi_{v}^{+}\right)^{-1}\left(x-d+\chi_{v}^{+}(\delta)\right) & \text { if } \chi_{v}^{+}\left(s_{0}\right)+d-\chi_{v}^{+}(\delta) \leq x \leq d .\end{cases}
$$


In [20], Lemmas 1-2, it was proved that $\chi_{v}^{+}$and $\Psi_{v}^{+}$are well-defined, absolutely continuous functions satisfying

$$
\begin{gathered}
\chi_{v}^{+}(\gamma)=c, \chi_{v}^{+}(\delta) \leq d, \quad\left(\chi_{v}^{+}\right)^{\prime}(\tau)>0 \text { for a.e. } \tau \in(\gamma, \delta) ; \\
\qquad d-\chi_{v}^{+}(\delta)=\operatorname{meas}\left(B_{v}\right) ; \\
\text { for a.e. } t \in A_{v} \text { there exists }\left(\chi_{v}^{+}\right)^{\prime}(v(t))=\frac{1}{v^{\prime}(t)} \\
\Psi_{v}^{+}(c)=\gamma, \Psi_{v}^{+}(d)=\delta,\left(\Psi_{v}^{+}\right)^{\prime}(x)>0 \text { for a.e. } x \notin\left(\chi_{v}^{+}\left(s_{0}\right), \chi_{v}^{+}\left(s_{0}\right)+d-\chi_{v}^{+}(\delta)\right) .
\end{gathered}
$$

In what follows we will use the following lemma.

Lemma 2.6. For a.e. $x \in\left[c, \chi_{v}^{+}(\delta)\right]$ such that there exists $\left(\Psi_{v}^{+}\right)^{\prime}(x)>0$, there exists a unique $t \in A_{v}$ such that

$$
\Psi_{v}^{+}(x)=v(t) \quad \text { and } \quad\left(\Psi_{v}^{+}\right)^{\prime}(x)=v^{\prime}(t) .
$$

Proof. In view of the definition of the function $\Psi_{v}^{+}$, it suffices to show that for a.e. $x \in\left[c, \chi_{v}^{+}(\delta)\right]$ there exists a unique $t \in A_{v}$ such that

$$
\left(\chi_{v}^{+}\right)^{-1}(x)=v(t) \quad \text { and } \quad\left(\left(\chi_{v}^{+}\right)^{-1}\right)^{\prime}(x)=v^{\prime}(t) .
$$

To this aim, first of all observe that the composition $g:=\chi_{v}^{+} \circ v$ is an increasing absolutely continuous function, defined in $[c, d]$ and assuming all the values of the interval $\left[c, \chi_{v}^{+}(\delta)\right]$.

Set $C_{v}:=\left\{t \in A_{v}:(2.10)\right.$ holds $\}$, we have that meas $\left(C_{v}\right)=$ meas $\left(A_{v}\right)$. Moreover, meas $\left(v\left(B_{v}\right)\right)=0$ and then by the absolute continuity of $\chi_{v}^{+}$we get meas $\left(g\left(B_{v}\right)\right)=0$. Hence, $\operatorname{meas}\left(g\left(C_{v}\right)\right)=\chi_{v}^{+}(\delta)-c$, that is for a.e. $x_{0} \in\left[c, \chi_{v}^{+}(\delta)\right]$ there exists a value $t_{0} \in C_{v}$ such that $x_{0}=g\left(t_{0}\right)=\chi_{v}^{+}\left(v\left(t_{0}\right)\right)$, i.e. $v\left(t_{0}\right)=\left(\chi_{v}^{+}\right)^{-1}\left(x_{0}\right)$. Moreover, by property $(2.10)$ we get that there exists $\left(\chi_{v}^{+}\right)^{\prime}\left(v\left(t_{0}\right)\right)=\frac{1}{v^{\prime}\left(t_{0}\right)}>0$, so there exists also the derivative of the inverse function $\left(\left(\chi_{v}^{+}\right)^{-1}\right)^{\prime}\left(\chi_{v}^{+}\left(v\left(t_{0}\right)\right)\right)=\left(\left(\chi_{v}^{+}\right)^{-1}\right)^{\prime}\left(x_{0}\right)=v^{\prime}\left(t_{0}\right)$.

Finally, since $v^{\prime}\left(t_{0}\right)>0$ and $v$ is increasing, we derive the uniqueness of the point $t_{0}$.

Analogous definitions and properties hold true when $v$ is decreasing. Indeed, let us define

$$
\chi_{v}^{-}(s):=d+\int_{\gamma}^{s}\left(w_{v}^{-}\right)^{\prime}(\sigma) \mathrm{d} \sigma, \quad \text { where } w_{v}^{-}(s):=\max \{x \in[c, d]: v(x)=s\}
$$

and

$$
\Psi_{v}^{-}(x):= \begin{cases}\left(\chi_{v}^{-}\right)^{-1}\left(x-c+\chi_{v}^{-}(\delta)\right) & \text { if } c \leq x \leq \chi_{v}^{-}\left(s_{0}\right)+c-\chi_{v}^{-}(\delta) \\ s_{0} & \text { if } \chi_{v}^{-}\left(s_{0}\right)+c-\chi_{v}^{-}(\delta) \leq x \leq \chi_{v}^{-}\left(s_{0}\right) \\ \left(\chi_{v}^{-}\right)^{-1}(x) & \text { if } \chi_{v}^{-}\left(s_{0}\right) \leq x \leq d\end{cases}
$$

with the same meaning for $s_{0}$ as above. It is easy to verify that making a change of variable, defining $v^{*}(\xi):=$ $v(d+c-\xi)$, one has

$$
w_{v}^{-}(s)=d+c-w_{v^{*}}^{+}(s), \quad \chi_{v}^{-}(s)=d+c-\chi_{v^{*}}^{+}(s), \quad \text { for every } s \in[\gamma, \delta]
$$

and $\Psi_{v}^{-}(x)=\Psi_{v^{*}}^{+}(d+c-x)$ for every $x \in[c, d]$. Therefore, we get

$$
\begin{gathered}
\chi_{v}^{-}(\gamma)=d, \chi_{v}^{-}(\delta) \geq c, \quad\left(\chi_{v}^{-}\right)^{\prime}(\tau)<0 \text { for a.e. } \tau \in(\gamma, \delta), \\
\chi_{v}^{-}(\delta)-c=\operatorname{meas}\left(B_{v}\right)
\end{gathered}
$$

and

$$
\Psi_{v}^{-}(c)=\delta, \Psi_{v}^{-}(d)=\gamma,\left(\Psi_{v}^{-}\right)^{\prime}(x)<0 \text { for a.e. } x \notin\left(\chi_{v}^{-}\left(s_{0}\right)+c-\chi_{v}^{-}(\delta), \chi_{v}^{-}\left(s_{0}\right)\right) .
$$

Moreover, a result analogous to Lemma 2.6 holds true. 
Now, if $v:[c, d] \rightarrow[\gamma, \delta]$ is a monotone function, we define

$$
\Psi_{v}(x):=\Psi_{v}^{+}(x) \quad \text { if } v \text { is increasing, } \quad \Psi_{v}(x):=\Psi_{v}^{-}(x) \quad \text { if } v \text { is decreasing. }
$$

By virtue of what we observed above, $\Psi_{v}$ is a monotone function such that $\Psi_{v}(c)=v(c), \Psi_{v}(d)=v(d)$ and

$$
\Psi_{v}(x)=s_{0} \quad \text { for a.e. } x \text { such that } \Psi_{v}^{\prime}(x)=0 .
$$

A key tool when dealing with relaxation problems is the Lyapunov Theorem for vector-valued functions (see e.g. [10], p. 1477). Its original formulation requires the summability of the involved functions, but it has been recently generalized in [20] to possibly non-summable functions. We state here a straightforward corollary of that result.

Theorem 2.7. Let $g_{j}: A \rightarrow[0,+\infty)^{m}, j=1, \ldots, h$, be vector-valued measurable functions on a set $A \subset \mathbb{R}$ with finite measure, and let $\lambda_{j}: A \rightarrow[0,1], j=1, \ldots, h$, be measurable weight functions with $\sum_{j=1}^{h} \lambda_{j}(x)=1$. Suppose that

$$
\sum_{j=1}^{h} \lambda_{j} g_{j} \in L^{1}\left(A ; \mathbb{R}^{m}\right) .
$$

Then, there exists a decomposition $E_{1}, \ldots, E_{h}$ of $A$ into disjoint measurable subsets such that $g_{j} \in L^{1}\left(E_{j} ; \mathbb{R}^{m}\right)$ for all $j$ and

$$
\sum_{j=1}^{h} \int_{E_{j}} g_{j}(x) \mathrm{d} x=\int_{A} \sum_{j=1}^{h} \lambda_{j}(x) g_{j}(x) \mathrm{d} x .
$$

\section{Monotonicity PRoperties}

The aim of this section is to investigate the monotonicity properties of the minimizers of $F$. More in detail, set

$$
\Upsilon^{*}:=\{v \in \Upsilon: \text { the following property }(*) \text { holds true }\}
$$

there exist $\tau_{1}, \tau_{2} \in[a, b], \tau_{1} \leq \tau_{2}$, such that $v$ is constant in $\left[\tau_{1}, \tau_{2}\right]$ and strictly monotone in $\left[a, \tau_{1}\right]$ and in $\left[\tau_{2}, b\right]$ with $v^{\prime} \neq 0$ a.e. in $\left[a, \tau_{1}\right] \cup\left[\tau_{2}, b\right]$.

The main tool for the results of this paper is the following.

Theorem 3.1. Let $f: I \times \mathbb{R} \rightarrow[0,+\infty)$ be a Borel-measurable function such that $f(\cdot, 0)$ is lower semicontinuous and satisfying (2.2) and (2.5). Then

$$
\inf _{\Upsilon} F=\inf _{\Upsilon *} F \quad \text { and } \quad \inf _{\Upsilon} F^{* *}=\inf _{\Upsilon *} F^{* *} .
$$

Moreover, if $\inf _{\Upsilon} F$ or $\inf _{\Upsilon} F^{* *}$ is attained, then there exists a minimizer in $\Upsilon^{*}$.

Proof. First we prove the result concerning the functional $F$. To this aim, notice that it suffices to show that for every $u \in \Upsilon$ and every $s_{0} \in u([a, b])$ such that $f\left(s_{0}, 0\right)=\min _{s \in u([a, b])} f(s, 0)$, there exists $w \in \Upsilon^{*}$ such that $w(x) \equiv s_{0}$ in $\left[\tau_{1}, \tau_{2}\right]$ and

$$
F(w) \leq F(u)
$$


To do this, note that by Lemma 2.2 we can assume without loss of generality that (2.4) holds true. Fix $u \in \Upsilon$ and choose $s_{0} \in u([a, b])$ such that $f\left(s_{0}, 0\right)=\min _{s \in u([a, b])} f(s, 0)$. By the lower semicontinuity of $f(\cdot, 0)$, there exist values $s_{0}$ as above. Now, we define $x_{1}, x_{2} \in[a, b], x_{1} \leq x_{2}, \xi_{1} \in\left[a, x_{1}\right]$ and $\xi_{2} \in\left[x_{2}, b\right]$ as follows:

$$
\begin{gathered}
x_{1}:=\min \left\{x \in[a, b]: u(x)=s_{0}\right\}, \quad x_{2}:=\max \left\{x \in[a, b]: u(x)=s_{0}\right\} ; \\
\xi_{1}:=\max \left\{x \in\left[a, x_{1}\right]: u(x)=\alpha\right\} \leq x_{1}, \quad \xi_{2}:=\min \left\{x \in\left[x_{2}, b\right]: u(x)=\beta\right\} \geq x_{2},
\end{gathered}
$$

and we consider the function $v:[a, b] \rightarrow u([a, b])$ defined by

$$
v(x):=\left\{\begin{array}{lll}
u\left(x-a+\xi_{1}\right) & \text { if } a \leq x \leq a+x_{1}-\xi_{1} \\
s_{0} & \text { if } a+x_{1}-\xi_{1} \leq x \leq b+x_{2}-\xi_{2} \\
u\left(x-b+\xi_{2}\right) & \text { if } b+x_{2}-\xi_{2} \leq x \leq b .
\end{array}\right.
$$

Observe that $v \in \Upsilon$ and $F(v) \leq F(u)$, indeed by (2.4) and the definition of $s_{0}$ we get

$$
\begin{aligned}
F(v)= & f\left(s_{0}, 0\right)\left[b+x_{2}-\xi_{2}-a-x_{1}+\xi_{1}\right]+\int_{a}^{a+x_{1}-\xi_{1}} f\left(u\left(x-a+\xi_{1}\right), u^{\prime}\left(x-a+\xi_{1}\right)\right) \mathrm{d} x \\
& +\int_{b+x_{2}-\xi_{2}}^{b} f\left(u\left(x-b+\xi_{2}\right), u^{\prime}\left(x-b+\xi_{2}\right)\right) \mathrm{d} x \\
= & \int_{a}^{\xi_{1}} f\left(s_{0}, 0\right) \mathrm{d} x+\int_{\xi_{1}}^{x_{1}} f\left(u(x), u^{\prime}(x)\right) \mathrm{d} x+\int_{x_{1}}^{x_{2}} f\left(s_{0}, 0\right) \mathrm{d} x+\int_{x_{2}}^{\xi_{2}} f\left(u(x), u^{\prime}(x)\right) \mathrm{d} x+\int_{\xi_{2}}^{b} f\left(s_{0}, 0\right) \mathrm{d} x \\
\leq & \int_{a}^{b} f\left(u(x), u^{\prime}(x)\right) \mathrm{d} x=F(u) .
\end{aligned}
$$

Moreover, note that

$$
\begin{aligned}
& v(a)=\alpha, v\left(a+x_{1}-\xi_{1}\right)=s_{0} \text { and } \min \left\{\alpha, s_{0}\right\}<v(x)<\max \left\{\alpha, s_{0}\right\} \text { for } a<x<a+x_{1}-\xi_{1} ; \\
& v\left(b+x_{2}-\xi_{2}\right)=s_{0}, v(b)=\beta \text { and } \min \left\{\beta, s_{0}\right\}<v(x)<\max \left\{\beta, s_{0}\right\} \text { for } b+x_{2}-\xi_{2}<x<b .
\end{aligned}
$$

Therefore, we can apply Lemma 2.5 and obtain that there exist two monotone functions $w_{1}:\left[a, a+x_{1}-\xi_{1}\right] \rightarrow \mathbb{R}$, $w_{2}:\left[b+x_{2}-\xi_{2}, b\right] \rightarrow \mathbb{R}$, such that

$$
\begin{gathered}
w_{1}(a)=v(a)=\alpha, w_{1}\left(a+x_{1}-\xi_{1}\right)=s_{0}, w_{2}\left(b+x_{2}-\xi_{2}\right)=s_{0}, w_{2}(b)=v(b)=\beta \\
w_{i}(x)=v(x), w_{i}^{\prime}(x)=v^{\prime}(x) \quad \text { for a.e. } x \text { such that } w_{i}^{\prime}(x) \neq 0, i=1,2 .
\end{gathered}
$$

Thus, let us define $w:[a, b] \rightarrow \mathbb{R}$ by

$$
w(x):= \begin{cases}\Psi_{w_{1}}(x) & \text { if } x \in\left[a, a+x_{1}-\xi_{1}\right] \\ s_{0} & \text { if } x \in\left[a+x_{1}-\xi_{1}, b+x_{2}-\xi_{2}\right] \\ \Psi_{w_{2}}(x) & \text { if } x \in\left[b+x_{2}-\xi_{2}, b\right]\end{cases}
$$

where $\Psi$ was defined in (2.14).

Of course, $w$ is absolutely continuous, with $w(a)=\alpha, w(b)=\beta$. Let us now show that $w \in \Upsilon^{*}$.

Indeed, observe that if $w_{1}$ is increasing then $s_{0} \geq \alpha$ and $w(x)=\Psi_{w_{1}}(x)=\Psi_{w_{1}}^{+}(x)$. Moreover, by definition (2.8) (taken for $c=a, d=a+x_{1}-\xi_{1}, \gamma=\alpha, \delta=s_{0}$ ), we have

$$
w(x)= \begin{cases}\left(\chi_{w_{1}}^{+}\right)^{-1}(x) & \text { if } a \leq x \leq \chi_{w_{1}}^{+}\left(s_{0}\right) \\ s_{0} & \text { if } \chi_{w_{1}}^{+}\left(s_{0}\right) \leq x \leq a+x_{1}-\xi_{1} .\end{cases}
$$


Instead, if $w_{1}$ is decreasing then $w(x)=\Psi_{w_{1}}(x)=\Psi_{w_{1}}^{-}(x)$. Moreover, $\alpha \geq s_{0}$ and by definition (2.13) (taken for $\left.c=a, d=a+x_{1}-\xi_{1}, \gamma=s_{0}, \delta=\alpha\right)$, we have $\chi_{w_{1}}^{-}\left(s_{0}\right)=a+x_{1}-\xi_{1}$ and

$$
w(x)= \begin{cases}\left(\chi_{w_{1}}^{-}\right)^{-1}\left(x-a+\chi_{w_{1}}^{-}(\alpha)\right) & \text { if } a \leq x \leq 2 a+x_{1}-\xi_{1}-\chi_{w_{1}}^{-}(\alpha) \\ s_{0} & \text { if } 2 a+x_{1}-\xi_{1}-\chi_{w_{1}}^{-}(\alpha) \leq x \leq a+x_{1}-\xi_{1} .\end{cases}
$$

Similar considerations for $w_{2}$ lead to deduce that

$$
w(x)= \begin{cases}s_{0} & \text { if } b+x_{2}-\xi_{2} \leq x \leq 2 b+x_{2}-\xi_{2}-\chi_{w_{2}}^{+}(\beta) \\ \left(\chi_{w_{2}}^{+}\right)^{-1}\left(x-d+\chi_{w_{2}}^{+}(\beta)\right) & \text { if } 2 b+x_{2}-\xi_{2}-\chi_{w_{2}}^{+}(\beta) \leq x \leq b ;\end{cases}
$$

if $w_{2}$ is increasing, whereas

$$
w(x)= \begin{cases}s_{0} & \text { if } b+x_{2}-\xi_{2} \leq x \leq \chi_{w_{2}}^{-}\left(s_{0}\right) \\ \left(\chi_{w_{2}}^{+}\right)^{-1}(x) & \text { if } \chi_{w_{2}}^{-}\left(s_{0}\right) \leq x \leq b\end{cases}
$$

if $w_{2}$ is decreasing. Therefore, $w \in \Upsilon^{*}$, that is there exist $\tau_{1} \in\left[a, \xi_{1}\right]$ and $\tau_{2} \in\left[\xi_{2}, b\right]$ such that $w(x)$ is strictly monotone in $\left[a, \tau_{1}\right]$, constant (equal to $s_{0}$ ) in $\left[\tau_{1}, \tau_{2}\right]$ and again strictly monotone in $\left[\tau_{2}, b\right]$.

It remains to prove that $F(w) \leq F(v)$. To this end, if $w_{1}$ is increasing let $h:\left[a, a+x_{1}-\xi_{1}\right] \rightarrow\left[a, \tau_{1}\right]$ be the function defined by $h(x):=\chi_{w_{1}}^{+}\left(w_{1}(x)\right)$. It is a strictly increasing absolutely continuous function, satisfying by $(2.10) h^{\prime}(x)=1$ for a.e. $x \in A_{w_{1}}:=\left\{x \in\left[a, a+x_{1}-\xi_{1}\right]: w_{1}^{\prime}(x)>0\right\}$. Therefore, defining

$$
g(x):= \begin{cases}f\left(\left(\chi_{w_{1}}^{+}\right)^{-1}(x),\left(\left(\chi_{w_{1}}^{+}\right)^{-1}\right)^{\prime}(x)\right) & \text { if } x \in A_{w_{1}} \\ 0 & \text { if } x \in\left[a, a+x_{1}-\xi_{1}\right] \backslash A_{w_{1}},\end{cases}
$$

we have that $g$ is a measurable non-negative function, hence we can apply the chain rule for absolutely continuous functions, and taking Lemma 2.5 into account, we obtain

$$
\begin{aligned}
\int_{a}^{\tau_{1}} f\left(\Psi_{w_{1}}(x), \Psi_{w_{1}}^{\prime}(x)\right) \mathrm{d} x & =\int_{a}^{\tau_{1}} g(x) \mathrm{d} x=\int_{a}^{a+x_{1}-\xi_{1}} g(h(t)) h^{\prime}(t) \mathrm{d} t \\
& =\int_{A_{w_{1}}} g(h(t)) \mathrm{d} t=\int_{A_{w_{1}}} f\left(w_{1}(t), w_{1}^{\prime}(t)\right) \mathrm{d} t=\int_{A_{w_{1}}} f\left(v(t), v^{\prime}(t)\right) \mathrm{d} t .
\end{aligned}
$$

By (2.9) and the definition of $s_{0}$ we deduce that setting $B_{w_{1}}:=\left\{x \in\left[a, a+x_{1}-\xi_{1}\right]: w_{1}^{\prime}(x)=0\right\}$, we have meas $\left(B_{w_{1}}\right)=a+x_{1}-\xi_{1}-\tau_{1}$. Therefore, by (2.4),

$$
\begin{aligned}
\int_{a}^{a+x_{1}-\xi_{1}} f\left(\Psi_{w_{1}}(x), \Psi_{w_{1}}^{\prime}(x)\right) \mathrm{d} x & =\int_{a}^{\tau_{1}} f\left(\Psi_{w_{1}}(x), \Psi_{w_{1}}^{\prime}(x)\right) \mathrm{d} x+f\left(s_{0}, 0\right)\left(a+x_{1}-\xi_{1}-\tau_{1}\right) \\
& \leq \int_{A_{w_{1}}} f\left(v(t), v^{\prime}(t)\right) \mathrm{d} t+\int_{B_{w_{1}}} f\left(v(t), v^{\prime}(t)\right) \mathrm{d} t=\int_{a}^{a+x_{1}-\xi_{1}} f\left(v(t), v^{\prime}(t)\right) \mathrm{d} t .
\end{aligned}
$$

An analogous argument works when $w_{1}$ is decreasing and similarly we can also deduce that

$$
\int_{b+x_{2}-\xi_{2}}^{b} f\left(\Psi_{w_{2}}(x), \Psi_{w_{2}}^{\prime}(x)\right) \mathrm{d} x \leq \int_{b+x_{2}-\xi_{2}}^{b} f\left(v(x), v^{\prime}(x)\right) \mathrm{d} x ;
$$

therefore $F(w) \leq F(v)$. Thus, the assertion is proved for $F$. 
Notice that we used the Borel measurability of $f$ only to guarantee that $F$ is well defined, i.e., that (2.6) holds, and this suffices to apply Lemma 2.2 (see Rem. 2.3). Therefore, in view of Lemma 2.1 the proof works also for $F^{* *}$.

Now we investigate more in detail what kind of monotonicity properties of the minimizers one can expect, according to the behavior of $f(\cdot, 0)$. To this purpose, let us split the class $\Upsilon^{*}$ into four subclasses as follows. Set

$$
\begin{aligned}
& \Upsilon_{M}:=\left\{v \in \Upsilon: \exists \tau_{1}, \tau_{2} \in(a, b) \text { with } \tau_{1} \leq \tau_{2}: v^{\prime}(x)<0 \text { a.e. in }\left(a, \tau_{1}\right),\right. \\
& \left.v^{\prime}(x)=0 \text { a.e. in }\left(\tau_{1}, \tau_{2}\right), v^{\prime}(x)>0 \text { a.e. in }\left(\tau_{2}, b\right)\right\} ; \\
& \Upsilon_{m}:=\left\{v \in \Upsilon: \exists \tau_{1}, \tau_{2} \in(a, b) \text { with } \tau_{1} \leq \tau_{2}: v^{\prime}(x)>0 \text { a.e. in }\left(a, \tau_{1}\right)\right. \text {, } \\
& \left.v^{\prime}(x)=0 \text { a.e. in }\left(\tau_{1}, \tau_{2}\right), v^{\prime}(x)<0 \text { a.e. in }\left(\tau_{2}, b\right)\right\} ; \\
& \Upsilon^{+}:=\left\{v \in \Upsilon: \exists \tau_{1}, \tau_{2} \in[a, b] \text { with } \tau_{1} \leq \tau_{2}: v^{\prime}(x)>0 \text { a.e. in }\left(a, \tau_{1}\right)\right. \text {, } \\
& \left.v^{\prime}(x)=0 \text { a.e. in }\left(\tau_{1}, \tau_{2}\right), v^{\prime}(x)>0 \text { a.e. in }\left(\tau_{2}, b\right)\right\} ; \\
& \Upsilon^{-}:=\left\{v \in \Upsilon: \exists \tau_{1}, \tau_{2} \in[a, b] \text { with } \tau_{1} \leq \tau_{2}: v^{\prime}(x)<0 \text { a.e. in }\left(a, \tau_{1}\right)\right. \text {, } \\
& \left.v^{\prime}(x)=0 \text { a.e. in }\left(\tau_{1}, \tau_{2}\right), v^{\prime}(x)<0 \text { a.e. in }\left(\tau_{2}, b\right)\right\} .
\end{aligned}
$$

Of course, $\Upsilon^{*}=\Upsilon_{M} \cup \Upsilon_{m} \cup \Upsilon^{+} \cup \Upsilon^{-}$. The class $\Upsilon_{M}$ collects the non-monotone trajectories of $\Upsilon^{*}$ satisfying the maximum principle (in the sense that their restriction on any subinterval attains maximum value at the boundary); similarly, the class $\Upsilon_{m}$ collects the non-monotone trajectories satisfying the minimum principle.

The next result can be immediately deduced in view of the proof of Theorem 3.1.

Corollary 3.2. Under the same assumptions of Theorem 3.1, let $\alpha \leq \beta$. Then in the statement of Theorem 3.1 the class $\Upsilon^{*}$ can be replaced by the class $\tilde{\Upsilon}$, according to various cases that can occur:

$$
\begin{aligned}
& \text { if } \min _{s \in[\alpha, \beta]} f(s, 0)=\inf _{s \in I} f(s, 0) \quad \Rightarrow \tilde{\Upsilon}:=\Upsilon^{+} ; \\
& \text {if } \min _{s \in[\alpha, \beta]} f(s, 0)=\inf _{s \in I, s \leq \beta} f(s, 0) \Rightarrow \tilde{\Upsilon}:=\Upsilon_{m} \cup \Upsilon^{+} ; \\
& \text {if } \min _{s \in[\alpha, \beta]} f(s, 0)=\inf _{s \in I, s \geq \alpha} f(s, 0) \Rightarrow \tilde{\Upsilon}:=\Upsilon_{M} \cup \Upsilon^{+} .
\end{aligned}
$$

Finally, if there exist $m_{1}:=\min _{s \leq \beta, s \in I} f(s, 0)$ and $m_{2}:=\min _{s \geq \alpha, s \in I} f(s, 0)$, setting

$$
s_{1}:=\max \left\{s \leq \beta: f(s, 0)=m_{1}\right\}, \quad s_{2}:=\min \left\{s \geq \alpha: f(s, 0)=m_{2}\right\},
$$

then the classes $\tilde{\Upsilon}$ above can be restricted to those collecting the trajectories $v$ taking values in $\left[\min \left\{\alpha, s_{1}\right\}\right.$, $\left.\max \left\{\beta, s_{2}\right\}\right]$.

Of course, an analogous result can be stated when $\alpha \geq \beta$, simply inverting them and replacing $\Upsilon^{+}$with $\Upsilon^{-}$.

Remark 3.3. Consider the constrained problem

$$
\operatorname{minimize}\left\{F(v)=\int_{a}^{b} f\left(v(t), v^{\prime}(t)\right) \mathrm{d} t: v \in \Upsilon^{+}\right\} .
$$

By Theorem 3.1 this problem is equivalent to minimize $F$ in the class

$$
\left\{v \in W^{1,1}(a, b): v(a)=\alpha, v(b)=\beta, v^{\prime}(x) \geq 0 \text { a.e. in }(a, b)\right\} .
$$


This variational problem has been investigated in [20]. By Corollary 3.2 if $\min _{s \in[\alpha, \beta]} f(s, 0)=\inf _{s \in I} f(s, 0)$, then the existence results in [20] still hold true for the free problem (2.1).

\section{Relaxation AND EXistence REsults}

In this section we investigate the relation between the solvability of (2.1) and of (2.3), under the condition $(2.2)$.

To this aim we will adopt the following notations.

For every $s \in I$ we set

$$
\begin{aligned}
\Delta_{s} & :=\left\{z \in \mathbb{R}: f^{* *}(s, z)<f(s, z)\right\}, \\
A^{+}(s) & :=\left\{z>0:(0, z) \subset \Delta_{s}\right\}, \\
A^{-}(s) & :=\left\{z<0:(z, 0) \subset \Delta_{s}\right\} .
\end{aligned}
$$

Moreover, let

$$
\phi^{+}(s):=\left\{\begin{array}{ll}
0 & \text { if } A^{+}(s)=\emptyset \\
\sup A^{+}(s) & \text { if } A^{+}(s) \neq \emptyset,
\end{array} \quad \phi^{-}(s):= \begin{cases}0 & \text { if } A^{-}(s)=\emptyset \\
\inf A^{-}(s) & \text { if } A^{-}(s) \neq \emptyset\end{cases}\right.
$$

In the sequel we will consider the following condition:

$$
\text { meas }\left(\left\{s \in[\alpha, \beta]: f^{* *}(s, 0)=\mu \text { and } \phi^{+}(s)>0 \text { or } \phi^{-}(s)<0\right\}\right)=0,
$$

where $\mu:=\min _{\sigma \in[\alpha, \beta]} f^{* *}(\sigma, 0)$.

Remark 4.1. Condition (4.2) is trivially satisfied if the restriction of $f^{* *}(\cdot, 0)$ to $[\alpha, \beta]$ has at most countable many minimizers or if $\phi^{ \pm}(s) \equiv 0$ in $[\alpha, \beta]$, that is when for every $s \in[\alpha, \beta]$ the detachment set $\Delta_{s}$ does not contain any interval $(-\delta, 0)$ or $(0, \delta)$.

Our first main result of this section is the following relaxation theorem.

Theorem 4.2. Let $f: I \times \mathbb{R} \rightarrow[0,+\infty)$ be a Borel-measurable function, with $f(\cdot, 0)$ lower semicontinuous, and such that the detachment set $\Delta_{s}$ is open for every $s \in I$. Suppose that (2.2), (2.5) and (4.2) hold true.

If (2.3) is solvable and admits a minimizer $v$ such that

$$
v^{\prime}(x) \in \operatorname{co}\left(\mathbb{R} \backslash \Delta_{v(x)}\right) \quad \text { for a.e. } x \in(a, b),
$$

then (2.1) is solvable too.

In the next result, we show that assumption (4.2) can be removed provided that $f(s, z)-f(s, 0)$ is coercive; i.e., there exists $\Phi:[0,+\infty) \rightarrow \mathbb{R}$ satisfying $\lim _{|z| \rightarrow+\infty} \frac{\Phi(|z|)}{|z|}=+\infty$, such that

$$
f(s, z)-f(s, 0) \geq \Phi(|z|) \forall s \in I \text { and } \forall z \in \mathbb{R} \text {. }
$$

Theorem 4.3. Let $f: I \times \mathbb{R} \rightarrow[0,+\infty)$ be a Borel-measurable function and let $f(\cdot, 0)$ be continuous. Let $(2.2)$, (2.5) and (4.4) be satisfied. Moreover, assume that $\Delta_{s}$ is open for every $s \in I$ and

$$
\phi^{+} \text {is lower semicontinuous and } \phi^{-} \text {is upper semicontinuous. }
$$

Then, if problem (2.3) is solvable, also problem (2.1) is solvable. 
The result below is the application of the above result to Lagrangians having an affine structure. It improves the analogous ones proved in [17,22] for integrands of sum type $g(s)+h(z)$.

Corollary 4.4. Let $f: I \times \mathbb{R} \rightarrow \mathbb{R}, f(s, z)=g(s)+\gamma(s) h(z)$. Assume that $g, \gamma: I \rightarrow[0,+\infty)$ are continuous, inf $\gamma>0$, and $h: \mathbb{R} \rightarrow[0,+\infty)$ is a lower semicontinuous function, coercive, satisfying $h(0)=h^{* *}(0)$. Then (2.1) is solvable.

To present the proof of Theorem 4.2 we need the following auxiliary result, which states that under certain conditions it is possible to modify the minimizer of the relaxed problem in such a way that its derivative is far from 0 in the intervals where the trajectory is strictly monotone.

Lemma 4.5. Let $f: I \times \mathbb{R} \rightarrow[0,+\infty)$ be a Borel-measurable function satisfying $(2.2)$ with $f(\cdot, 0)$ lower semicontinuous and assume that (2.5) and (4.2) hold true.

If problem (2.3) is solvable, then there exists a minimizer $v \in \Upsilon^{*}$ such that

$$
\begin{aligned}
& v^{\prime}(x) \geq \phi^{+}(v(x)) \text { for a.e. } x \text { such that } v^{\prime}(x)>0 \\
& v^{\prime}(x) \leq \phi^{-}(v(x)) \text { for a.e. } x \text { such that } v^{\prime}(x)<0
\end{aligned}
$$

Proof. By Theorem 3.1, since (2.3) is solvable, there exists a solution $v$ belonging to $\Upsilon^{*}$.

Assume firstly that $v^{\prime}(x)>0$ a.e. in $\left(a, \tau_{1}\right), v^{\prime}(x)<0$ a.e. in $\left(\tau_{2}, b\right)$. Recall that we can choose the value $s_{0}$ in such a way that

and consequently,

$$
s_{0}:=\min \left\{\sigma \in v([a, b]): f^{* *}(\sigma, 0)=\min _{s \in v([a, b])} f^{* *}(s, 0)\right\}
$$

$$
f^{* *}(v(x), 0)>f^{* *}\left(s_{0}, 0\right) \quad \text { for every } x \in\left[a, \tau_{1}\right) \cup\left(\tau_{2}, b\right] .
$$

Observe now that the restriction of $v$ to the interval $\left(a, \tau_{1}\right)$ is a minimizer of the constrained problem

$$
\operatorname{minimize} \int_{a}^{\tau_{1}} f^{* *}\left(u(x), u^{\prime}(x)\right) \mathrm{d} x: u \in \Upsilon_{0}^{+}
$$

where

$$
\Upsilon_{0}^{+}:=\left\{u \in W^{1,1}\left(a, \tau_{1}\right): u(a)=\alpha, u\left(\tau_{1}\right)=s_{0}, u^{\prime}(x) \geq 0 \text { a.e. in }\left(a, \tau_{1}\right)\right\} .
$$

Hence, by [20], Theorem 7 and Remark 4, $v$ satisfies the following DuBois-Reymond condition

$$
f^{* *}\left(v(x), v^{\prime}(x)\right)-c \in v^{\prime}(x) \partial f^{* *}\left(v(x), v^{\prime}(x)\right) \quad \text { a.e. in }\left(a, \tau_{1}\right)
$$

for some constant $c \leq f^{* *}\left(s_{0}, 0\right)$.

For every $s$ there exist $q(s)$ and $m(s)$ such that

$$
f^{* *}(s, z)=q(s)+m(s) z \quad \text { for every } z \in\left[0, \phi^{+}(s)\right] .
$$

From the previous relation written for $z=0$ we deduce $q(s)=f^{* *}(s, 0)$, so we can write

$$
f^{* *}(s, z)=f^{* *}(s, 0)+m(s) z \quad \text { for every } z \in\left[0, \phi^{+}(s)\right] .
$$

Thus, by (4.9) we deduce the existence of a constant $c \leq f^{* *}\left(s_{0}, 0\right)$ such that

$$
f^{* *}(v(x), 0)+m(v(x)) v^{\prime}(x)-c=v^{\prime}(x) m(v(x)) \quad \text { for a.e. } x \in\left(a, \tau_{1}\right) \text { with } v^{\prime}(x)<\phi^{+}(v(x)) .
$$

Hence by (4.8) we get

$$
f^{* *}\left(s_{0}, 0\right)<f^{* *}(v(x), 0)=c \leq f^{* *}\left(s_{0}, 0\right) \quad \text { for a.e. } x \in\left(a, \tau_{1}\right) \text { such that } v^{\prime}(x)<\phi^{+}(v(x)),
$$


thus

$$
v^{\prime}(x) \geq \phi^{+}(v(x)) \text { for a.e. } x \in\left(a, \tau_{1}\right) .
$$

The same argument works in $\left(\tau_{2}, b\right)$ and one deduces that $v^{\prime}(x) \leq \phi^{-}(v(x))$ for a.e. $x \in\left(\tau_{2}, b\right)$.

The case $v^{\prime}(x)<0$ a.e. in $\left(a, \tau_{1}\right)$ and $v^{\prime}(x)>0$ a.e. in $\left(\tau_{2}, b\right)$ can be treated similarly to the previous one, taking

$$
s_{1}:=\max \left\{\sigma \in v([a, b]): f^{* *}(\sigma, 0)=\min _{s \in v([a, b])} f^{* *}(s, 0)\right\}
$$

instead of $s_{0}$, in such a way that (4.8) holds again.

Finally, let us consider the case when $v$ is monotone increasing, with $v^{\prime}(x)>0$ a.e. in $\left(a, \tau_{1}\right), v(x) \equiv s_{0}$ in $\left(\tau_{1}, \tau_{2}\right), v^{\prime}(x)>0$ a.e. in $\left(\tau_{2}, b\right)$. The same argument as above shows that $v^{\prime}(x) \geq \phi^{+}(v(x))$ for a.e. $x \in\left(a, \tau_{1}\right)$, so let us consider the interval $\left(\tau_{2}, b\right)$.

Let $w$ denote the restriction of $v$ in $\left(\tau_{2}, b\right)$; then since $w^{\prime}(x)>0$ a.e. we derive that its inverse function $w^{-1}$ is absolutely continuous too (see $e . g$. [2]). Hence, by (4.2) we have

$$
\begin{aligned}
\operatorname{meas}\left(\left\{x \in\left(\tau_{2}, b\right): f^{* *}(v(x), 0)=\mu \text { and } v^{\prime}(x)<\phi^{+}(v(x))\right\}\right) \\
\quad \leq \operatorname{meas}\left(\left\{x \in\left(\tau_{2}, b\right): f^{* *}(v(x), 0)=\mu \text { and } 0<\phi^{+}(v(x))\right\}\right) \\
\quad=\operatorname{meas}\left(w^{-1}\left(\left\{s \in\left(s_{0}, \beta\right): f^{* *}(s, 0)=\mu \text { and } \phi^{+}(s)>0\right\}\right)\right)=0 .
\end{aligned}
$$

Hence, repeating the argument above, starting from the DuBois-Reymond condition (4.9), we deduce that

$$
f^{* *}\left(s_{0}, 0\right)<f^{* *}(v(x), 0)=c \leq f^{* *}\left(s_{0}, 0\right) \quad \text { for a.e. } x \in\left(\tau_{2}, b\right) \text { such that } v^{\prime}(x)<\phi^{+}(v(x)),
$$

thus

$$
v^{\prime}(x) \geq \phi^{+}(v(x)) \text { for a.e. } x \in\left(\tau_{2}, b\right) .
$$

A straightforward consequence of the lemma above is the following non-existence result.

Proposition 4.6. Suppose that the assumptions of Lemma 4.5 hold true. If $\alpha<\beta$ and $\phi^{+}(s) \equiv+\infty$, then $(2.3)$ has no solution. The same conclusion holds if $\alpha>\beta$ and $\phi^{-}(s) \equiv-\infty$.

Now, we are ready to prove our first relaxation theorem.

Proof of Theorem 4.2. Let $v$ be a minimizer of (2.3). By Theorem 3.1 we can assume that $v \in \Upsilon^{*}$. Moreover, if $s_{0}$ is as in (4.7), we can assume that $v(x) \equiv s_{0}$ in $\left[\tau_{1}, \tau_{2}\right]$ and $v$ is strictly monotone in $\left(a, \tau_{1}\right)$ and in $\left(\tau_{2}, b\right)$, with $v^{\prime}(x) \neq 0$ almost everywhere.

Finally, in view of the proof of Theorem 3.1, by Lemmas 2.5 and 2.6, we can also assume that condition (4.3) holds true for the modified minimizer too.

Let us now consider the interval $\left(a, \tau_{1}\right)$ (supposing it to be non-degenerate) and assume that $v^{\prime}(x)>0$ a.e. in $\left(a, \tau_{1}\right)$ with $v\left(\tau_{1}\right)=s_{0}$ (the proof is analogous if $v$ is decreasing in $\left[a, \tau_{1}\right]$ ).

By Lemma 4.5 we can also assume that $v^{\prime}(x) \geq \phi^{+}(v(x))$ for a.e. $x$ such that $v^{\prime}(x)>0$. So, by definition of $\phi^{+}$and by virtue of (4.3) the functions

$$
\begin{gathered}
\xi_{1}(x):=\sup \left\{z: z \leq v^{\prime}(x) \text { and } f(v(x), z)=f^{* *}(v(x), z)\right\}, \\
\xi_{2}(x):=\inf \left\{z: z \geq v^{\prime}(x) \text { and } f(v(x), z)=f^{* *}(v(x), z)\right\}
\end{gathered}
$$

are well-defined and positive. Moreover, since $\Delta_{s}$ is open for every $s \in[\alpha, \beta]$, we have

$$
f\left(v(x), \xi_{i}(x)\right)=f^{* *}\left(v(x), \xi_{i}(x)\right) \quad \text { for every } x \in\left(a, \tau_{1}\right), i=1,2
$$

and $f^{* *}(v(x), \cdot)$ is affine in $\left[\xi_{1}(x), \xi_{2}(x)\right]$ (notice that this interval can be degenerate). 
We claim that $\xi_{1}$ and $\xi_{2}$ are measurable functions. Indeed, as for $\xi_{1}(x)$, let us define $g:[a, b] \times \mathbb{R} \rightarrow[0,+\infty)$ by

$$
g(x, z):=f(u(x), z)-f^{* *}(u(x), z)+\left(z-u^{\prime}(x)\right)_{+}
$$

where $(a)_{+}$denotes the positive part of the real number $a$. By Lemma 2.1, now applied to the Borel function $(x, z) \mapsto f(u(x), z)$ in place of $(s, z) \mapsto f(s, z)$, we get that $x \mapsto f^{* *}(u(x), z)$ is Lebesgue-measurable for all $z$. Then $(x, z) \mapsto-f^{* *}(u(x), z)+\left(z-u^{\prime}(x)\right)_{+}$is a Carathéodory function so, by [16], Proposition VIII.1.1, there exists a Borel function $h:[a, b] \times \mathbb{R} \rightarrow[0,+\infty)$, such that $h(x, z)=-f^{* *}(u(x), z)+\left(z-u^{\prime}(x)\right)_{+}$for almost all $x$ and every $z \in \mathbb{R}$. Therefore, the function $\tilde{g}:[a, b] \times \mathbb{R} \rightarrow[0,+\infty)$, defined by $\tilde{g}(x, z):=f(u(x), z)+h(x, z)$, is Borel-measurable and

$$
g(x, \cdot)=\tilde{g}(x, \cdot) \quad \text { for almost all } x .
$$

As in the proof of Lemma 2.1, since the set $B_{k}:=\left\{(x, z) \in(\tilde{g})^{-1}(\{0\}): z>k\right\}$ is Borel-measurable for every $k$, then $\pi\left(B_{k}\right)$ is Lebesgue-measurable, where $\pi$ is the projection $\pi(x, z):=x$. As it is easy to verify, for every $k \in \mathbb{R}$ we have

$$
\left\{x \in[a, b]: \xi_{1}(x)>k\right\}=\pi\left(\left\{(x, z) \in g^{-1}(\{0\}): z>k\right\}\right),
$$

and the last set is equal almost everywhere to $\pi\left(B_{k}\right)$, because of (4.12). Hence $\xi_{1}$ is Lebesgue-measurable. The proof for $\xi_{2}$ is analogous.

Set

$$
\psi_{i}(s):=\frac{1}{\xi_{i}\left(v^{-1}(s)\right)}, \quad \text { for } s \in\left(\alpha, s_{0}\right), i=1,2 .
$$

We claim that $\psi_{1}, \psi_{2}$ are measurable functions. Indeed, again by [16], Proposition VIII.1.1, there exists Borel functions $\tilde{\xi}_{1}, \tilde{\xi}_{2}$ such that $\tilde{\xi}_{i}(x)=\xi_{i}(x)$ for a.e. $x \in(a, b), i=1,2$. Set $\tilde{\psi}_{i}(s):=1 / \tilde{\xi}_{i}\left(v^{-1}(s)\right), i=1,2$. Since $v^{-1}$ is continuous, then $\tilde{\psi}_{i}(s)$ is Borel-measurable for $i=1,2$. Moreover, set $A_{i}:=\left\{x: \xi_{i}(x) \neq \tilde{\xi}_{i}(x)\right\}$, we have $v\left(A_{i}\right)=\left\{s: \psi_{i}(s) \neq \tilde{\psi}_{i}(s)\right\}$ and by the absolute continuity of $v$ we get meas $\left(v\left(A_{i}\right)\right)=0, i=1,2$. Therefore, the functions $\psi_{i}$ are Lebesgue-measurable, $i=1,2$.

Notice that since $\xi_{1}(x) \leq v^{\prime}(x) \leq \xi_{2}(x)$, we have $\psi_{2}(s) \leq \frac{1}{v^{\prime}\left(v^{-1}(s)\right)} \leq \psi_{1}(s)$, hence there exists a measurable weight function $\lambda:\left(\alpha, s_{0}\right) \rightarrow[0,1]$ such that

$$
\frac{1}{v^{\prime}\left(v^{-1}(s)\right)}=\lambda(s) \psi_{1}(s)+(1-\lambda(s)) \psi_{2}(s) .
$$

Hence, in particular, the map $s \mapsto \lambda(s) \psi_{1}(s)+(1-\lambda(s)) \psi_{2}(s)$ is summable in $\left(\alpha, s_{0}\right)$.

Let us define $\tilde{f}, \tilde{f^{* *}}: I \times(0,+\infty) \rightarrow \mathbb{R}$ as

$$
\tilde{f}(s, z):=f\left(s, \frac{1}{z}\right) z, \quad \tilde{f^{*} *}(s, z):=f^{* *}\left(s, \frac{1}{z}\right) z .
$$

In [20], Lemma 5 , it was proved that $f^{* *}(s, \cdot)$ is affine in $(c, d), c>0$, if and only if $f^{* *}(s, \cdot)$ is affine in $\left(\frac{1}{d}, \frac{1}{c}\right)$. Therefore, by (4.11) we deduce that

$$
\tilde{f}\left(s, \psi_{i}(s)\right)=\tilde{f^{* *}}\left(s, \psi_{i}(s)\right) \quad \text { a.e. in }\left(\alpha, s_{0}\right), i=1,2
$$

and $\tilde{f}^{* *}(s, \cdot)$ is affine in $\left[\psi_{2}(s), \psi_{1}(s)\right]$. Hence, by $(4.13)$

$$
\tilde{f^{\tilde{*} *}}\left(s, \frac{1}{v^{\prime}\left(v^{-1}(s)\right)}\right)=\lambda(s) \tilde{f^{* *}}\left(s, \psi_{1}(s)\right)+(1-\lambda(s)) \tilde{f^{\tilde{*} *}}\left(s, \psi_{2}(s)\right)
$$

so, in particular, also the map $s \mapsto \lambda(s) \tilde{f^{* *}}\left(s, \psi_{1}(s)\right)+(1-\lambda(s)) \tilde{f^{* *}}\left(s, \psi_{2}(s)\right)$ is summable in $\left(\alpha, s_{0}\right)$, being

$$
\int_{\alpha}^{s_{0}} f^{\tilde{*} *}\left(s, \frac{1}{v^{\prime}\left(v^{-1}(s)\right)}\right) \mathrm{d} s=\int_{a}^{\tau_{1}} f^{* *}\left(v(x), v^{\prime}(x)\right) \mathrm{d} x \leq F^{* *}(v) .
$$


Thus, we can apply Theorem 2.7 to the functions $g_{i}(s):=\left(\psi_{i}(s), f^{* *}\left(s, \psi_{i}(s)\right)\right), i=1,2$, deducing the existence of a decomposition of $\left(\alpha, s_{0}\right)$ into disjoint measurable subsets $F_{1}, F_{2}$ such that, if we define $\gamma(s):=$ $\psi_{1}(s) \chi_{F_{1}}(s)+\psi_{2}(s) \chi_{F_{2}}(s)$, then by (4.13) we have

$$
\int_{\alpha}^{s_{0}} \gamma(s) \mathrm{d} s=\int_{\alpha}^{s_{0}} \frac{1}{v^{\prime}\left(v^{-1}(s)\right)} \mathrm{d} s
$$

and by (4.14), (4.15)

$$
\begin{aligned}
\int_{\alpha}^{s_{0}} \tilde{f}(s, \gamma(s)) \mathrm{d} s & =\int_{F_{1}} \tilde{f * *}\left(s, \psi_{1}(s)\right) \mathrm{d} s+\int_{F_{2}} \tilde{f * *}\left(s, \psi_{2}(s)\right) \mathrm{d} s \\
& =\int_{\alpha}^{s_{0}}\left[\lambda(s) \tilde{f^{* *}}\left(s, \psi_{1}(s)\right)+(1-\lambda(s)) \tilde{f^{*} *}\left(s, \psi_{2}(s)\right)\right] \mathrm{d} s=\int_{\alpha}^{s_{0}} \tilde{f * *}\left(s, \frac{1}{v^{\prime}\left(v^{-1}(s)\right)}\right)
\end{aligned}
$$

Consider

$$
U(s):=a+\int_{\alpha}^{s} \gamma(\sigma) \mathrm{d} \sigma
$$

we have that $U$ is an absolutely continuous function, with $U(\alpha)=a$, and by (4.16)

$$
U\left(s_{0}\right)=a+\int_{\alpha}^{s_{0}} \gamma(s) \mathrm{d} s=a+\int_{\alpha}^{s_{0}} \frac{1}{v^{\prime}\left(v^{-1}(s)\right)} \mathrm{d} s=a+\int_{a}^{\tau_{1}} \frac{1}{v^{\prime}(x)} v^{\prime}(x) \mathrm{d} x=\tau_{1} .
$$

Finally, since $U(s)$ is absolutely continuous with $U^{\prime}(s)>0$ a.e. in $\left[\alpha, s_{0}\right]$, then also the inverse function $\hat{v}(x):=U^{-1}(x), x \in\left[a, \tau_{1}\right]$, is absolutely continuous (see e.g. [2]). Moreover, $\hat{v}(a)=\alpha$ and $\hat{v}\left(\tau_{1}\right)=s_{0}$ and finally by $(4.17)$

$$
\begin{aligned}
\int_{a}^{\tau_{1}} f\left(\hat{v}(x), \hat{v}^{\prime}(x)\right) \mathrm{d} x & =\int_{\alpha}^{s_{0}} f\left(s, \hat{v}^{\prime}(U(s))\right) U^{\prime}(s) \mathrm{d} s=\int_{\alpha}^{s_{0}} \tilde{f}(s, \gamma(s)) \mathrm{d} s \\
& =\int_{\alpha}^{s_{0}} \tilde{f * *}\left(s, \frac{1}{v^{\prime}\left(v^{-1}(s)\right)}\right) \mathrm{d} s=\int_{a}^{\tau_{1}} f^{* *}\left(v(x), v^{\prime}(x)\right) \mathrm{d} x .
\end{aligned}
$$

A similar construction can be made in the interval $\left[\tau_{2}, b\right]$ so that the modified trajectory $\hat{v}$ (kept equal to $s_{0}$ in $\left.\left[\tau_{1}, \tau_{2}\right]\right)$ satisfies the following chain of inequalities

$$
F(\hat{v})=\int_{a}^{b} f^{* *}\left(v(x), v^{\prime}(x)\right) \mathrm{d} x \leq \int_{a}^{b} f^{* *}\left(w(x), w^{\prime}(x)\right) \mathrm{d} x \leq F(w)
$$

for every trajectory $w \in \Upsilon$, that is $\hat{v}$ is a minimizer of $(2.1)$.

Remark 4.7. By the proof of the above result and Lemma 4.5, we have that if the assumptions of Theorem 4.2 hold true and if there exists a solution $v$ to (2.3) satisfying (4.3), then there exists a solution $w$ to (2.1) satisfying (4.6).

Remark 4.8. A variant in the definition of $\phi^{ \pm}$can be considered. For every $s \in I$ let us denote

$$
\tilde{A}^{+}(s):=\left\{z>0: f^{* *}(s, \cdot) \text { is affine in }(0, z)\right\}, \tilde{A}^{-}(s):=\left\{z<0: f^{* *}(s, \cdot) \text { is affine in }(z, 0)\right\}
$$

and define

$$
\tilde{\phi}^{+}(s):=\left\{\begin{array}{ll}
\sup \tilde{A}^{+}(s) & \text { if } \tilde{A}^{+}(s) \neq \emptyset \\
0 & \text { if } \tilde{A}^{+}(s)=\emptyset,
\end{array} \quad \tilde{\phi}^{-}(s):= \begin{cases}\inf \tilde{A}^{-}(s) & \text { if } \tilde{A}^{-}(s) \neq \emptyset \\
0 & \text { if } \tilde{A}^{-}(s)=\emptyset .\end{cases}\right.
$$

With this change (to be done also in the definition of (4.2)) Theorem 4.2 still holds true. We choose definition (4.1) for $\phi^{ \pm}$in order to have a weaker condition (4.2). 
Remark 4.9. If $\alpha=\beta$ then (4.2) is trivially satisfied.

In order to prove Theorem 4.3 we use an argument similar to that presented in [17], Theorem 6 , based upon the approximation result [17], Lemma 5, stated below, for the sake of completeness.

Lemma 4.10. Let $g: \mathbb{R} \rightarrow \mathbb{R}$ be a lower semicontinuous function. Then there exists a sequence $\left(g_{n}\right)$ of $C^{1}(\mathbb{R})$ functions such that:

(i) $g_{n}(s) \rightarrow g(s)$ for every $s \in \mathbb{R}$;

(ii) for each $n$ and each interval $[\gamma, \delta]$

$$
\left\{s \in[\gamma, \delta]: g_{n}^{\prime}(s)=0\right\} \text { is finite; }
$$

(iii) for each interval $[\gamma, \delta]$ there exists $n_{0}$ such that

$$
g_{n}(s) \leq g_{n+1}(s) \text { for all } s \in[\gamma, \delta] \text { and all } n>n_{0} .
$$

Moreover, if $g$ is bounded below then also the sequence $\left(g_{n}\right)$ is equibounded below.

Proof of Theorem 4.3. Define $g: \mathbb{R} \rightarrow[0,+\infty), g(s):=f(s, 0)$ if $s \in I$ and extended by lower semicontinuity on $\mathbb{R} \backslash I$, so to be constant on the connected components of $\mathbb{R} \backslash I$. Let $g_{n}(s)$ be as in Lemma 4.10 . Since $g \geq 0$ then there exists $L \in \mathbb{R}$ such that $g_{n}(s) \geq L$ for all $s$ and for all $n$.

For all $n$, consider $h_{n}: I \times \mathbb{R} \rightarrow \mathbb{R}$,

$$
h_{n}(s, z):=f(s, z)-f(s, 0)+g_{n}(s)
$$

so that

$$
h_{n}^{* *}(s, z)=f^{* *}(s, z)-f(s, 0)+g_{n}(s) .
$$

As it is well known (see e.g. [10], Thm. 10.3.i), the function $\Phi$ in (4.4) can be assumed to be convex, so that

$$
h_{n}^{* *}(s, z) \geq \Phi(|z|)+g_{n}(s) \geq \Phi(|z|)+L .
$$

Moreover, by Lemma 2.1 the function $s \mapsto h_{n}^{* *}(s, z)$ is Lebesgue-measurable on $I$, for every $z \in \mathbb{R}$. Hence, by [15], Theorem 1, the functional

$$
H_{n}^{* *}(v)=\int_{a}^{b} h_{n}^{* *}\left(v(t), v^{\prime}(t)\right) \mathrm{d} t, \quad v \in \Upsilon
$$

is well defined and lower semicontinuous. Thus, by the direct method of the Calculus of Variations, for every $n \in \mathbb{N}$ there exists a solution $u_{n}$ to

$$
\operatorname{minimize} H_{n}^{* *}(v)=\left\{\int_{a}^{b} h_{n}^{* *}\left(v(t), v^{\prime}(t)\right) \mathrm{d} t: v \in \Upsilon\right\}
$$

Notice that

$$
\left\{z \in \mathbb{R}: f^{* *}(s, z)<f(s, z)\right\}=\left\{z \in \mathbb{R}: h_{n}^{* *}(s, z)<h_{n}(s, z)\right\}
$$

so that (2.2) and Lemma 4.10 (ii) imply

$$
\operatorname{meas}\left(\left\{s \in[\alpha, \beta]: h_{n}^{* *}(s, 0)=\min _{\sigma \in[\alpha, \beta]} h_{n}^{* *}(\sigma, 0)\right\}\right)=\operatorname{meas}\left(\left\{s \in[\alpha, \beta]: g_{n}(s)=\min _{\sigma \in[\alpha, \beta]} g_{n}(\sigma)\right\}\right)=0 \text {. }
$$


Thus, by Lemma 4.6, we may assume that $u_{n} \in \Upsilon^{*}$ and

$$
\begin{aligned}
& u_{n}^{\prime}(x) \geq \phi^{+}\left(u_{n}(x)\right) \text { for a.e. } x \text { such that } u_{n}^{\prime}(x)>0 \\
& u_{n}^{\prime}(x) \leq \phi^{-}\left(u_{n}(x)\right) \text { for a.e. } x \text { such that } u_{n}^{\prime}(x)<0 .
\end{aligned}
$$

More precisely, since each $u_{n}$ is in $\Upsilon^{*}$, there exist $a_{n}, b_{n}, c_{n}$ and $d_{n}$ in $[a, b], a_{n} \leq b_{n} \leq c_{n} \leq d_{n}$, such that

$$
\begin{array}{ll}
u_{n}^{\prime}(x) \leq \phi^{-}\left(u_{n}(x)\right) & \text { for a.e. } x \in\left[a, a_{n}\right] \\
u_{n}^{\prime}(x) \geq \phi^{+}\left(u_{n}(x)\right) & \text { for a.e. } x \in\left[a_{n}, b_{n}\right] \\
u_{n}^{\prime}(x)=0 & \text { for a.e. } x \in\left[b_{n}, c_{n}\right] \\
u_{n}^{\prime}(x) \geq \phi^{+}\left(u_{n}(x)\right) & \text { for a.e. } x \in\left[c_{n}, d_{n}\right] \\
u_{n}^{\prime}(x) \leq \phi^{-}\left(u_{n}(x)\right) & \text { for a.e. } x \in\left[d_{n}, b\right] .
\end{array}
$$

Fix $\bar{u} \in \Upsilon$ such that $F^{* *}(\bar{u})<+\infty$ and let $\bar{J}:=\bar{u}([a, b])$, compact sub-interval of $I$. By Lemma 4.10 (iii) there exists $\bar{n}$ such that

$$
g_{n}(s) \leq g_{n+1}(s) \text { for all } s \in \bar{J} \text { and all } n>\bar{n} .
$$

Therefore, by (4.18), the minimality of $u_{n},(4.21)$ and Lemma 4.10 (i) we have that for all $n>\bar{n}$

$$
\int_{a}^{b}\left(\Phi\left(\left|u_{n}^{\prime}(x)\right|\right)+L\right) \mathrm{d} x \leq H_{n}^{* *}\left(u_{n}\right) \leq H_{n}^{* *}(\bar{u}) \leq \int_{a}^{b} f^{* *}\left(\bar{u}(x), \bar{u}^{\prime}(x)\right) \mathrm{d} x<+\infty .
$$

So, we can assume, possibly up to subsequences, that there exists $u_{0} \in \Upsilon$ and points $a^{\prime}, b^{\prime}, c^{\prime}, d^{\prime}$ in $[a, b]$ such that $u_{n} \rightarrow u_{0}$ in $W^{1,1}(a, b), a_{n} \rightarrow a^{\prime}, b_{n} \rightarrow b^{\prime}, c_{n} \rightarrow c^{\prime}, d_{n} \rightarrow d^{\prime}$. In particular, up to subsequences, $u_{n}$ pointwise converges to $u_{0}$ and $u_{n}^{\prime}$ weakly converges to $u_{0}^{\prime}$ in $L^{1}$.

We claim that

$$
\begin{array}{ll}
u_{0}^{\prime}(x) \leq \phi^{-}\left(u_{0}(x)\right) & \text { for a.e. } x \in\left[a, a^{\prime}\right] \\
u_{0}^{\prime}(x) \geq \phi^{+}\left(u_{0}(x)\right) & \text { for a.e. } x \in\left[a^{\prime}, b^{\prime}\right] \\
u_{0}^{\prime}(x)=0 & \text { for a.e. } x \in\left[b^{\prime}, c^{\prime}\right] \\
u_{0}^{\prime}(x) \geq \phi^{+}\left(u_{0}(x)\right) & \text { for a.e. } x \in\left[c^{\prime}, d^{\prime}\right] \\
u_{0}^{\prime}(x) \leq \phi^{-}\left(u_{0}(x)\right) & \text { for a.e. } x \in\left[d^{\prime}, b\right] .
\end{array}
$$

Indeed, as for the first inequality, if $u_{0}^{\prime}(x)-\phi^{-}\left(u_{0}(x)\right)>0$ in a subset $C \subset\left[a, a^{\prime}\right]$ having positive measure, then there exists an integer $\bar{n}$ such that for every $n \geq \bar{n}$ we have $a_{n}>a^{\prime}-\frac{1}{2}$ meas $(C)$. Therefore, setting $\tilde{C}:=C \backslash\left[a^{\prime}-\frac{1}{2} \operatorname{meas}(C), a^{\prime}\right]$ we have meas $(\tilde{C})>0$ and $\tilde{C} \subset\left[a, a_{n}\right]$ for every $n \geq \bar{n}$. Then, by (4.20), using the weak convergence of $\left(u_{n}^{\prime}\right)_{n}$, the Fatou Lemma and the upper semicontinuity of $\phi^{-}$, we get

$$
\begin{aligned}
0 & \geq \liminf _{n \rightarrow+\infty} \int_{\tilde{C}}\left(u_{n}^{\prime}(x)-\phi^{-}\left(u_{n}(x)\right)\right) \mathrm{d} x=\int_{\tilde{C}} u_{0}^{\prime}(x) \mathrm{d} x-\limsup _{n \rightarrow+\infty} \int_{\tilde{C}} \phi^{-}\left(u_{n}(x)\right) \mathrm{d} x \\
& \geq \int_{\tilde{C}} u_{0}^{\prime}(x) \mathrm{d} x-\int_{\tilde{C}} \limsup _{n \rightarrow+\infty} \phi^{-}\left(u_{n}(x)\right) \mathrm{d} x \geq \int_{\tilde{C}}\left(u_{0}^{\prime}(x)-\phi^{-}\left(u_{0}(x)\right)\right) \mathrm{d} x>0,
\end{aligned}
$$

which is absurd, and the first inequality of (4.23) is proved. The other inequalities can be proved in a similar way.

By (4.22) and taking the coercivity of $\Phi$ into account, we have that $\left(\left|u_{n}^{\prime}\right|\right)_{n>\bar{n}}$ is bounded in $L^{1}(a, b)$, then there exists a compact interval $[A, B]$ including $u_{n}([a, b])$ for all $n>\bar{n}$.

By Lemma 4.10 (iii) there exists $n_{0} \geq \bar{n}$ such that

$$
g_{n}(s) \leq g_{n+1}(s) \quad \text { for all } s \in[A, B] \text { and all } n>n_{0} .
$$


By the convexity of $h_{n}^{* *}(s, \cdot)$, for all $k>n_{0}$ we get

$$
\begin{aligned}
\liminf _{n \rightarrow+\infty} H_{n}^{* *}\left(u_{n}\right) & \geq \liminf _{n \rightarrow+\infty} \int_{a}^{b} f^{* *}\left(u_{n}(x), u_{n}^{\prime}(x)\right) \mathrm{d} x+\liminf _{n \rightarrow+\infty} \int_{a}^{b}\left(g_{n}\left(u_{n}(x)\right)-f\left(u_{n}(x), 0\right)\right) \mathrm{d} x \\
& \geq \int_{a}^{b} f^{* *}\left(u_{0}(x), u_{0}^{\prime}(x)\right) \mathrm{d} x+\liminf _{n \rightarrow+\infty} \int_{a}^{b}\left(g_{k}\left(u_{n}(x)\right)-f\left(u_{n}(x), 0\right)\right) \mathrm{d} x .
\end{aligned}
$$

By the continuity of $g_{k}$ and $f(\cdot, 0)$

$$
\liminf _{n \rightarrow+\infty} \int_{a}^{b}\left(g_{k}\left(u_{n}(x)\right)-f\left(u_{n}(x), 0\right)\right) \mathrm{d} x \geq \int_{a}^{b}\left(g_{k}\left(u_{0}(x)\right)-f\left(u_{0}(x), 0\right)\right) \mathrm{d} x
$$

and the last term goes to 0 as $k$ goes to $+\infty$, due to Lemma 4.10 (i). Thus,

$$
\liminf _{n \rightarrow+\infty} H_{n}^{* *}\left(u_{n}\right) \geq F^{* *}\left(u_{0}\right) .
$$

Now, fix $v \in \Upsilon$. By Lemma 4.10 (iii) applied with $[c, d]=v\left([a, b]\right.$ ) and by the minimality of $u_{n}$

$$
F^{* *}(v)=\lim _{n \rightarrow+\infty} H_{n}^{* *}(v) \geq \liminf _{n \rightarrow+\infty} H_{n}^{* *}\left(v_{n}\right) .
$$

So, we have proved that $u_{0}$ is a solution to (2.3), satisfying (4.23). Thanks to (4.23) we can avoid the use of Lemma 4.5 in the proof of Theorem 4.2. Since assumption (4.2) was used only to apply such a lemma, and since, by (4.4), for every $s \in I$

$$
\operatorname{co}\left\{z \in \mathbb{R}: f^{* *}(s, z)=f(s, z)\right\}=\operatorname{co}\left\{z \in \mathbb{R}: f^{* *}(s, z)-f(s, 0)=f(s, z)-f(s, 0)\right\}=\mathbb{R}
$$

so that $u_{0}$ satisfies (4.3), we obtain the assertion.

We conclude this section with the proof of the relaxation result for coercive Lagrangians of affine type.

Proof of Corollary 4.4. It is a straightforward consequence of Theorem 4.3. In fact, by Lemma 2.2, without loss of generality we can assume that $h(z) \geq h(0)$ for all $z$. Then

$$
f(s, z)-f(s, 0)=\gamma(s)[h(z)-h(0)] \geq(\inf \gamma)[h(z)-h(0)]
$$

and condition (4.4) is satisfied. Moreover, since $f$ has the affine structure with inf $\gamma>0$, then $\Delta_{s}$ does not depend on $s$ and is open.

Remark 4.11. We note that results similar to some of those stated in this section appeared, in the coercive setting, in two papers by Ornelas (see $[23,24]$ ), but they are based on some preliminary results announced without proof, referred back to preprints by the same author, never appeared or available in any form to the scientific community. In particular, the proof of a property called by the author derivative bounded away from 0 , analogous to that proved in the present Lemma 4.5, was never published, according to our knowledge.

\section{NeCESSARY CONDItions AND NON-EXISTENCE RESUlts}

In this section, we establish the validity of the DuBois-Reymond necessary condition expressed in terms of a differential inclusion involving the subdifferential of $f(s, \cdot)$ in the sense of Convex Analysis, even if $f(s, \cdot)$ is non-convex in general.

From now on we need to strengthen slightly the regularity assumptions on the function $f$. Throughout this section we will assume $f(\cdot, 0)$ continuous and $f(s, \cdot)$ continuous at $z=0$ for every $s \in I$. 
In [12] the validity of the DuBois-Reymond condition was proved under a certain monotonicity assumption on $f(\cdot, 0)$ (see [12], Thm. 4.1 and $(\mathrm{H} 3)$ ). Such a result is based upon [12], Theorem 3.1, which has the same assertion of Theorem 3.1 here proved. It is easy to see that with no change in the proofs, just using the present Theorem 3.1 and taking account of [21], Remark 4, the result holds true also in the present more general setting, stated as follows.

Theorem 5.1. Let $f: I \times \mathbb{R} \rightarrow[0,+\infty)$ be Borel-measurable, with $f(\cdot, 0)$ continuous and $f(s, \cdot)$ continuous at $z=0$ for every $s \in I$. Suppose that (2.2) and (2.5) hold true.

If (2.1) is solvable then there exists a solution $u \in \Upsilon^{*}$ such that $\partial f\left(u(x), u^{\prime}(x)\right) \neq \emptyset$ for a.e. $x \in(a, b)$ and the following DuBois-Reymond condition holds

$$
f\left(u(x), u^{\prime}(x)\right)-c \in u^{\prime}(x) \partial f\left(u(x), u^{\prime}(x)\right) \quad \text { a.e. in }(a, b)
$$

for some constant $c \leq \min _{s \in u([a, b])} f(s, 0)$.

Moreover, if $u^{\prime}(x)=0$ in a set having positive measure then $c=\min _{s \in u([a, b])} f(s, 0)$.

Hence, $f\left(u(x), u^{\prime}(x)\right)=f^{* *}\left(u(x), u^{\prime}(x)\right)$ for a.e. $x$ in $(a, b)$, and $\partial f\left(u(x), u^{\prime}(x)\right)=\partial f^{* *}\left(u(x), u^{\prime}(x)\right)$ for a.e. $x$ in $(a, b)$. In particular,

$$
f^{* *}\left(u(x), u^{\prime}(x)\right)-c \in u^{\prime}(x) \partial f^{* *}\left(u(x), u^{\prime}(x)\right) \quad \text { for a.e. } x \in(a, b),
$$

for some constant $c \leq \min _{s \in u([a, b])} f(s, 0)$.

The limitation on the constant $c$ in condition (5.1) allows to derive the following non-existence result, which is the analogous of [12], Proposition 5.1.

Proposition 5.2. Let $\alpha \leq \beta$. Under the same assumptions of Theorem 5.1, suppose further that

$$
\operatorname{ess} \sup _{s \in[\alpha, \beta]} \liminf _{|z| \rightarrow+\infty} \inf _{t \in \partial f^{* *}(s, z)}\left(f^{* *}(s, z)-z t\right)>\min _{s \in[\alpha, \beta]} f(s, 0) .
$$

Then, problem (2.1) does not admit solution.

Proof. The proof goes as that of the analogous result in [12], but now we use Theorem 5.1 in place of [12], Theorem 4.4. Nevertheless we write it for the sake of completeness.

First observe that by the convexity of $f^{* *}$ we have that the function

$$
g_{s}(z):=\inf _{t \in \partial f^{* *}(s, z)}\left(f^{* *}(s, z)-z t\right)
$$

is increasing in $(-\infty, 0)$ and decreasing in $(0,+\infty)$, for every $s \in I$. Indeed, if $z_{2} \geq z_{1} \geq 0$ and $\xi \in \partial f^{* *}\left(s, z_{2}\right)$, we have $f^{* *}\left(s, z_{1}\right)-f^{* *}\left(s, z_{2}\right) \geq \xi\left(z_{1}-z_{2}\right)$ and $\xi \geq \sup \partial f^{* *}\left(s, z_{1}\right)$; so, since $z_{1} \geq 0$ we get

$$
g_{s}\left(z_{1}\right)=f^{* *}\left(s, z_{1}\right)-z_{1} \sup \partial f^{* *}\left(s, z_{1}\right) \geq f^{* *}\left(s, z_{1}\right)-z_{1} \xi \geq f^{* *}\left(s, z_{2}\right)-z_{2} \xi \geq g_{s}\left(z_{2}\right) .
$$

The proof of the monotonicity in $(-\infty, 0)$ proceeds similarly. As a consequence, $\liminf _{|z| \rightarrow+\infty} g_{s}(z)=\inf _{z \in \mathbb{R}} g_{s}(z)$. So, by (5.3) there exists a set $H \subset[\alpha, \beta]$ having positive measure such that

$$
\inf _{t \in \partial f^{* *}(s, z)}\left(f^{* *}(s, z)-z t\right)>\min _{s \in[\alpha, \beta]} f(s, 0) \quad \text { for every } s \in H, z \in \mathbb{R} .
$$

Assume by contradiction that (2.1) admits a solution. Then, by Theorem 5.1 there exists an optimal trajectory $u$ satisfying the condition (5.2) for some constant $c \leq \min _{s \in u([a, b])} f(s, 0)$. Put $A:=\{x \in[a, b]: u(x) \in H\}$, 
we have meas $(A)>0$ since $u$ is absolutely continuous. Hence

$$
c \in f^{* *}\left(u(x), u^{\prime}(x)\right)-u^{\prime}(x) \partial f^{* *}\left(u(x), u^{\prime}(x)\right) \quad \text { for some } x \in A
$$

and this implies

$$
\inf _{t \in \partial f^{* *}\left(u(x), u^{\prime}(x)\right)}\left(f^{* *}\left(u(x), u^{\prime}(x)\right)-u^{\prime}(x) t\right) \leq \min _{s \in[\alpha, \beta]} f(s, 0) \quad \text { for some } x \in A
$$

in contradiction with $(5.4)$.

The previous result finds a simple immediate application to the relevant case of integrands having the affine structure

$$
f(s, z)=g(s)+\gamma(s) h(z)
$$

Set

$$
\ell:=\liminf _{|z| \rightarrow+\infty} \inf _{t \in \partial h^{* *}(z)}\left(h^{* *}(z)-z t\right)
$$

The following result holds.

Proposition 5.3. Let $g, \gamma: I \rightarrow[0,+\infty)$ be continuous functions. Let $h: \mathbb{R} \rightarrow[0,+\infty)$ be lower semicontinuous, continuous at 0 and such that $h(0)=h^{* *}(0)$. Suppose moreover that $\ell>-\infty$.

Then, if

$$
\max _{s \in[\alpha, \beta]}\{g(s)+\gamma(s) \ell\}>\min _{s \in[\alpha, \beta]}\{g(s)+\gamma(s) h(0)\}
$$

problem (2.1) has no solution.

Proof. First note that the assumptions of Theorem 5.1 are trivially satisfied.

Let $\bar{s} \in[\alpha, \beta]$ be such that

$$
g(\bar{s})+\gamma(\bar{s}) \ell=\max _{s \in[\alpha, \beta]}\{g(s)+\gamma(s) \ell\} .
$$

If (5.5) holds, by the continuity of $g$ and $\gamma$ we have $g(s)+\gamma(s) \ell>\min _{s \in[\alpha, \beta]}\{g(s)+\gamma(s) h(0)\}$ for every $s$ in a neighborhood of $\bar{s}$. Since, by definition of $\ell$,

$$
\begin{aligned}
\liminf _{|z| \rightarrow+\infty} \inf _{t \in \partial f^{* *}(s, z)}\left(f^{* *}(s, z)-z t\right) & =g(s)+\gamma(s) \liminf _{|z| \rightarrow+\infty} \inf _{t \in \partial h^{* *}(z)}\left(h^{* *}(z)-z t\right) \\
& =g(s)+\gamma(s) \ell
\end{aligned}
$$

then we have that (5.3) holds. By Proposition 5.2 we deduce the conclusion.

Example 5.1. Let $f(s, z):=\gamma(s)\left(\sqrt{1+z^{2}}+k\right)$, with $\gamma$ continuous and non-negative and $k>0$. In this case it is easy to check that $\ell=k$, so if $k \max _{s \in[\alpha, \beta]} \gamma(s)>(k+1) \min _{s \in[\alpha, \beta]} \gamma(s)$, problem (2.1) does not admit solution.

Example 5.2. Let $f(s, z):=g(s)+\gamma(s)|z|$, with $g, \gamma$ continuous and non-negative. In this case $\ell=h(0)=0$, so if $g$ is not constant then problem (2.1) has no solution.

Example 5.3. Let $f(s, z):=g(s)+\gamma(s) \sqrt{1+z^{2}}$, with $g, \gamma$ continuous and non-negative. In this case $\ell=0$, so if $\max _{s \in[\alpha, \beta]} g(s)>\min _{s \in[\alpha, \beta]}\{g(s)+\gamma(s)\}$, problem $(2.1)$ does not admit solution.

Acknowledgements. The authors thank an anonymous referee for pointing out the paper [15], that allowed the proof of Lemma 2.1. 


\section{REFERENCES}

[1] L. Ambrosio, O. Ascenzi and G. Buttazzo, Lipschitz regularity for minimizers of integral functionals with highly discontinuous integrands. J. Math. Anal. Appl. 142 (1989) 301-316.

[2] V.I. Bogachev, Measure Theory, Volume I. Springer-Verlag, Berlin, Germany (2007).

[3] B. Botteron and B. Dacorogna, Existence of solutions for a variational problem associated to models in optimal foraging theory. J. Math. Anal. Appl. 147 (1990) 263-276.

[4] B. Botteron and B. Dacorogna, Existence and nonexistence results for noncoercive variational problems and applications in ecology. J. Differ. Equ. 85 (1990) 214-235.

[5] B. Botteron and P. Marcellini, A general approach to the existence of minimizers of one-dimensional noncoercive integrals of the calculus of variations. Ann. Inst. Henri Poincaré, Anal. non linéaire 8 (1991) 197-223.

[6] P. Celada and S. Perrotta, Existence of minimizers for nonconvex, noncoercive simple integrals. SIAM J. Control Optim. 41 (2002) 1118-1140.

[7] A. Cellina, The classical problem of the calculus of variations in the autonomous case: relaxation and lipschitzianity of solutions. Trans. Amer. Math. Soc. 356 (2004) 415-426.

[8] A. Cellina and A. Ferriero, Existence of Lipschitzian solutions to the classical problem of the calculus of variations in the autonomous case. Ann. Inst. Henri Poincaré, Anal. non linéaire 20 (2003) 911-919.

[9] A. Cellina, G. Treu and S. Zagatti, On the minimum problem for a class of non-coercive functionals. J. Differ. Equ. 127 (1996) $225-262$.

[10] L. Cesari, Optimization: theory and applications. Springer-Verlag, New York, USA (1983).

[11] F.H. Clarke, An indirect method in the calculus of variations. Trans. Amer. Math. Soc. 336 (1993) 655-673.

[12] G. Cupini, M. Guidorzi and C. Marcelli, Necessary conditions and non-existence results for autonomous nonconvex variational problems. J. Differ. Equ. 243 (2007) 329-348.

[13] B. Dacorogna, Direct methods in the Calculus of Variations, Applied Mathematical Sciences 78. Second edition, Springer, Berlin, Germany (2008).

[14] G. Dal Maso and H. Frankowska, Autonomous integral functionals with discontinuous nonconvex integrands: Lipschitz regularity of minimizers, DuBois-Reymond necessary conditions, and Hamilton-Jacobi equations. Appl Math Optim. 48 (2003) $39-66$.

[15] E. De Giorgi, G. Buttazzo and G. Dal Maso, On the lower semicontinuity of certain integral functionals. Atti Accad. Naz. Lincei, VIII. Ser. 74 (1983) 274-282.

[16] I. Ekeland and R. Temam, Convex analysis and variational problems, Studies in Mathematics and its Applications 1. North Holland, Amsterdam, The Netherlands (1976).

[17] N. Fusco, P. Marcellini and A. Ornelas, Existence of minimizers for some nonconvex one-dimensional integrals. Port. Math. 55 (1998) 167-185.

[18] O.A. Ladyzhenskaya and N.N. Ural'tseva, Linear and Quasilinear Elliptic Equations, Mathematics in Science and Engineering 46. Academic Press, New York-London (1968).

[19] C. Marcelli, Variational problems with nonconvex, noncoercive, highly discontinuous integrands: characterization and existence of minimizers. SIAM J. Control Optim. 40 (2002) 1473-1490.

[20] C. Marcelli, Necessary and sufficient conditions for optimality of nonconvex, noncoercive autonomous variational problems with constraints. Trans. Amer. Math. Soc. 360 (2008) 5201-5227.

[21] P. Marcellini, Alcune osservazioni sull'esistenza del minimo di integrali del calcolo delle variazioni senza ipotesi di convessità. Rend. Mat. Appl. 13 (1980) 271-281.

[22] A. Ornelas, Existence of scalar minimizers for nonconvex simple integrals of sum type. J. Math. Anal. Appl. 221 (1998) $559-573$.

[23] A. Ornelas, Existence and regularity for scalar minimizers of affine nonconvex simple integrals. Nonlinear Anal. 53 (2003) $441-451$.

[24] A. Ornelas, Existence of scalar minimizers for simple convex integrals with autonomous Lagrangian measurable on the state variable. Nonlinear Anal. 67 (2007) 2485-2496.

[25] J.P. Raymond, Existence and uniqueness results for minimization problems with nonconvex functionals. J. Optim. Theory Appl. 82 (1994) 571-592. 